\title{
THE QUANTUM ENTROPY AS AN ULTIMATE VISITING CARD OF THE DE BROGLIE-BOHM THEORY
}

\section{FISCALETTI}

PACS 03.65.-w; 03.65.Ta; 03.65.Ud; 04.60.-m (c) 2012

The de Broglie-Bohm theory is an interesting approach to quantum mechanics, which has the merit to describe atomic and subatomic processes without ascribing a special role to the observer and remaining faithful to the principle of causality and the motion dogma. In this article, a new suggestive interpretation of the de Broglie-Bohm theory is proposed. It is based on the idea that the quantum entropy is its ultimate visiting card in the quantum domain, in a relativistic curved space-time, and in the quantum gravity domain.

\section{Introduction}

Quantum mechanics is, perhaps, the fundamental physical theory of the 20-th century, which has determined the most profound changes in the image of the world. In order to explain atomic and subatomic processes, quantum mechanics - as it has been formulated by its founders, the representatives of the Copenhagen and Göttingen schools (Bohr, Heisenberg, Born, etc...) threw out two essential classical concepts, the principle of causality and the dogma of formulation of physics in terms of motion in space-time (motion dogma). According to the Copenhagen interpretation of quantum mechanics, it is not possible to provide a causal description of microscopic processes: the wave function carries only the information about possible outcomes of a measurement process. Whenever a measurement is performed, the wave function ceases to evolve according to the Schrödinger equation (the evolution law of every isolated microscopic system) and collapses into one of its eigenstates. The absolute squares of the scalar products of the wave function with its eigenfunctions are the probabilities (or probability densities) of the occurrence of these particular eigenvalues in the measurement process $[1,2]$.

The central claim made by the standard quantum theory is that the wave function provides a complete description of a quantum system: all we can know about a physical system is contained in its wave function. This would seem to imply that quantum mechanics is, funda- mentally, a theory of wave functions. However, accepting such a proposition faces us with an inescapable problem, most eloquently formulated by Schrödinger within the context of his famous cat paradox: namely, the question of what it actually means for an object to literally exist in a superposition of eigenstates of the measurement operator [3].

The Copenhagen interpretation of quantum mechanics can be considered as the "minimal" interpretation of quantum theory: it provides us only the mathematical structure and the minimum interpretative propositions needed to define the relation between the mathematical structure itself and the experience. Although it functions perfectly under the point of view of the empirical predictions, it is clearly characterized by inner contradictions and cannot be considered completely self-consistent. In synthesis: many authors do not find satisfactory the resort to two different postulates as regards the modality of evolution of a system whether it is subjected to the observation or not; that, in virtue of the unlimited validity of the superposition principle, superpositions of macroscopic states such as alive cat - dead cat, according to the famous Schrödinger's mental experiment, exist; that a boundary between the microscopic world (governed by the superposition principle) and the macroscopic world (in which we have well-defined perceptions as regards the properties of physical systems) cannot be defined in a precise way [4]. The failure of the orthodox quantum theory to offer any sort of coherent resolution to concerns of this sort is largely the reason for which it has continually remained so ambiguous and obscure.

On the basis of all these considerations, since the birth of quantum theory renowned physicists such as Einstein, Planck, Schrödinger, de Broglie did not accept the acausal interpretation of quantum mechanics proposed by the Copenhagen and Göttingen schools and tried to find alternative interpretations. The de Broglie-Bohm theory (sometimes referred to as Bohmian mechanics or pilot-wave theory), originally proposed by Louis de Broglie in 1927 for one-body systems and then extended by David Bohm in 1952 also to the treatment of many- 
body systems, allows us to accomplish all of the perplexities listed above in the simplest way. The essential insight of the de Broglie-Bohm theory is that quantum mechanics is, fundamentally, a theory about particles in motion and, thus, that in addition to the wave function, the description of a quantum system should also include its configuration - that is to say, the precise positions of all the particles of the system at all times. What naturally results from this insight is a deterministic quantum theory of particle trajectories: a theory, predictably equivalent to quantum mechanics, which allows us to give a causal completion to quantum mechanics and to explain the quantum behavior of matter remaining faithful to the principle of causality and the motion dogma, which not only accommodates, but provides the simplest known explanation for the quantum formalism (including the Born rule, the Heisenberg uncertainty relation, the representation of dynamical variables as Hilbert space operators, and so on). The de Broglie-Bohm version of quantum mechanics reproduces all the empirical results of quantum theory and, at the same time, has the merit to describe atomic and subatomic processes without ascribing a crucial role to the observer and to recover some causality also in the microscopic world that is so seen as a world, in which quantum particles have precise trajectories [5-8]. The Bohmian mechanics constitutes, thus, a quantum theory without observers, i.e., a theory that is formulated not in terms of what observers see but in terms of objective events, regardless of whether or not they are observed. In the Bohmian mechanics, the role of the wave function is to tell the matter how to move. According to this theory, all the quantum behaviors of matter (which appear enigmatic, mysterious, and exotic in the standard interpretation) result from a self-interaction of the particle. Each particle which exerts a quantum force on itself can be expressed in terms of a quantum potential derived from the particle wave function.

The purpose of this article is to provide a new suggestive reading of the de Broglie-Bohm theory, in which a physical quantity appropriately called quantum entropy can be considered as a fundamental physical entity, the ultimate grid, the real ultimate "visiting card" of quantum mechanics. The paper is structured in the following manner. In Section 2, we will review the fundamental features of the non-relativistic de Broglie-Bohm theory. In Section 3, we will define the quantum entropy and will outline the perspectives introduced by the quantum entropy in the non-relativistic de Broglie-Bohm theory. In Section 4, we will extend the approach of the quantum entropy in the relativistic de Broglie-Bohm theory in a curved space-time. In Section 5, we will analyze the perspectives of the quantum entropy in the Bohmian quantum gravity.

\section{About the Non-Relativistic de Broglie-Bohm Theory and the Quantum Potential}

The de Broglie-Bohm theory is based on two fundamental starting hypotheses. Before all, the idea that quantum mechanics is not complete and must be completed by adding supplementary parameters to the formalism, the so-called hidden variables. The hidden variables of the model are the positions of all the particles constituting the physical system into examination. The first starting hypothesis can be expressed so: the physical system is prepared in such a way that, at the initial time $t=0$, it is associated with a specific wave function $\psi(\mathbf{x}, 0)$, which is assumed to be known perfectly and, moreover, is at a point $\mathbf{x}$ (among those compatible with the wave function into examination) that instead we ignore (it is in this sense that the position is a hidden variable of this theory).

The second starting hypothesis of the Bohmian mechanics is de Broglie's objective wave-corpuscle dualism. On the ground of this idea originally proposed by de Broglie in 1926 at the Solvay Conference, each fundamental particle of physics is assumed to be constituted by a corpuscle and by a wave which surrounds it and accompanies it during its motion. As regards the nonrelativistic problem, de Broglie suggested that the wave function of such an object was associated with a set of identical particles which have different positions and are distributed in space according to the usual quantum formula, given by $|\psi(\mathbf{x})|^{2}$. But he recognized a dual role for the wave function: on one side, it determines the probable position of the particle (just like in the standard interpretation); on the other side, it influences the position by exerting a force on the orbit. According to de Broglie's approach, the wave function would act like a sort of pilot wave which guides the particles in regions, where such wave function is more intense $[9,10]$.

Bohm's version of quantum mechanics is practically the de Broglie pilot-wave theory carried to its logical conclusion. In his classic works in 1952, Bohm succeeded in developing a mathematical treatment of de Broglie's objective wave-particle dualism and thus was the first to realize that this theory provided a foundation for nonrelativistic quantum mechanics. He showed that if one interprets each individual physical system as that composed by a corpuscle and a wave guiding it, by writing its wave function in polar form and decomposing the 
Schrödinger equation, the movement of the corpuscle under the guide of the wave happens in agreement with a law of motion, which assumes the following form

$\frac{\partial S}{\partial t}+\frac{|\nabla S|^{2}}{2 m}-\frac{\hbar^{2}}{2 m} \frac{\nabla^{2} R}{R}+V=0$

(where $R$ is the amplitude, and $S$ is the phase of the wave function, $\hbar$ is Planck's reduced constant, $m$ is the mass of the particle and $V$ is the classical potential). This equation is equal to the classical equation of HamiltonJacobi except for the appearance of the additional term

$Q=-\frac{\hbar^{2}}{2 m} \frac{\nabla^{2} R}{R}$

having the dimension of an energy and containing Planck's constant and, therefore, the appropriately defined the quantum potential [11]. Taking into account that the quantity $-\frac{\partial S}{\partial t}$ is the total energy of the particle and that $\frac{|\nabla S|^{2}}{2 m}$ is its kinetic energy, Eq. (1) can also be written in the convenient form

$\frac{|\nabla S|^{2}}{2 m}-\frac{\hbar^{2}}{2 m} \frac{\nabla^{2} R}{R}+V=-\frac{\partial S}{\partial t}$,

which can be seen as a real energy conservation law in quantum mechanics. From Eq. (3), one can deduce immediately that, without the quantum potential (2), the total energy of the system would not be conserved: this means that the quantum potential plays an essential role in the quantum formalism.

The equation of motion (1) of a particle can also be expressed in the equivalent form

$m \frac{d^{2} \mathbf{x}}{d t^{2}}=-\nabla(V+Q)$

where $\mathbf{x}=\mathbf{x}(t)$ is the trajectory of the particle associated with its wave function. Equation (4) has just the form of Newton's second law of classical mechanics, in which the particle is subjected to a quantum force (derived from the quantum potential) in addition to a classical force (derived from a classical potential). Therefore, we can say that, according to the de Broglie-Bohm theory, each subatomic particle is completely described by its wave function, which evolves according to the usual Schrödinger equation

$i \hbar \frac{\partial \psi}{\partial t}=H \psi$

and its configuration and follows a precise trajectory $\mathbf{x}=\mathbf{x}(t)$ in space-time that is originated by the action of a classical potential and a quantum potential (and that evolves according to Eq. (1) or the equivalent equation (4)). Moreover, it is important to underline that, in the Bohmian mechanics, the decomposition of the Schrödinger equation (5) leads, besides to the quantum Hamilton-Jacobi equation (1), also to a continuity equation for the probability density $\rho(\mathbf{x}, t)=R^{2}(\mathbf{x}, t)=$ $|\psi(\mathbf{x}, t)|^{2}$ :

$-\frac{\partial \rho}{\partial t}=\nabla \cdot\left(\rho \frac{\nabla S}{m}\right)$.

Equation (6) says that, in the de Broglie-Bohm theory, all individual trajectories demonstrate the collective behavior like a liquid flux [12], perhaps, a superconductive one.

The treatment provided by relations (1)-(4) and (6) can be extended in a simple way to many-body systems. If we consider a wave function $\psi=$ $R\left(\mathbf{x}_{1}, \ldots, \mathbf{x}_{N}, t\right) e^{i S\left(\mathbf{x}_{1}, \ldots, \mathbf{x}_{N}, t\right) / \hbar}$, defined on the configuration space $R^{3 N}$ of a system of $N$ particles, the movement of this system under the action of the wave $\psi$ happens in agreement to the law of motion

$\frac{\partial S}{\partial t}+\sum_{i=1}^{N} \frac{\left|\nabla_{i} S\right|^{2}}{2 m_{i}}+Q+V=0$

where

$Q=\sum_{i=1}^{N}-\frac{\hbar^{2}}{2 m_{i}} \frac{\nabla_{i}^{2} R}{R}$

is the many-body quantum potential. Equation (7) can also be written in the convenient form

$\sum_{i=1}^{N} \frac{\left|\nabla_{i} S\right|^{2}}{2 m_{i}}+Q+V=-\frac{\partial S}{\partial t}$,

which can be considered as a real energy conservation law in quantum mechanics for a many-body system. The equation of motion of the $i$-th particle, in the limit of big separations, can also be written in the form

$m_{i} \frac{\partial^{2} \mathbf{x}_{i}}{\partial t^{2}}=-\left[\nabla_{i} Q\left(\mathbf{x}_{1}, \mathbf{x}_{2}, \ldots, \mathbf{x}_{n}\right)+\nabla_{i} V_{i}\left(\mathbf{x}_{i}\right)\right]$,

which is a quantum Newton law for a many-body system. Equation (10) shows that the contribution to the total force acting on the $i$-th particle coming from the quantum potential, i.e. $\nabla_{i} Q$, is a function of the positions of all the other particles and, thus, does not decrease in general, as the distance increases. The continuity equation for the probability density becomes

$-\frac{\partial \rho}{\partial t}=\sum_{i=1}^{N} \nabla_{i} \cdot\left(\rho \frac{\nabla_{i} S}{m}\right)$. 
In synthesis, the Bohmian mechanics claims that, in our world, electrons and other elementary particles have precise positions at every time $t$ and follow precise trajectories that evolve according to Eqs. (1), (3), and (4) (for a one-body system) or according to Eqs. (7), (9), and (10) (for a many-body system). This picture turns out to be in contrast with the standard interpretation of quantum mechanics, according to which the quantum particles do not have precise positions, but are regarded as "delocalized" to the extent, to which the wave function is spread out. It is also in contrast with another picture of the Bohmian trajectories, that one often has in mind when using Bohmian trajectories for numerical purposes, namely the hydrodynamic picture. According to the latter, all the Bohmian trajectories associated with a given wave function (but corresponding to a different initial position) are on an equal footing, none is more real than the others, they are all regarded as flow lines in analogy to the flow lines of a classical fluid. In the Bohmian mechanics, instead, only one of the Bohmian trajectories corresponds to reality, and all the other ones are no more than mathematical curves, representing possible alternative histories that could have occurred if the initial configuration of our world had been different, but did not occur [13].

The most important and relevant element in order to understand the features of Bohm's version of quantum mechanics is certainly the quantum potential. In virtue of the features of the quantum potential, the basic equations (1) and (4) (or the equivalent equations (7) and (10) for a many-body system) of the non-relativistic de Broglie-Bohm theory do not imply a classical treatment of quantum processes [14]. The quantum potential does not have the usual properties expected from a classical potential. On the basis of its definition (2) (or the analogous relation (8) for a many-body system), the quantum potential depends on how the amplitude of the wave function varies in space. The presence of the Laplace operator indicates that the action of this potential is likespace, namely creates a non-local instantaneous action onto the particle under consideration. The appearance of the amplitude of the wave function in the denominator also explains why the quantum potential can produce strong long-range effects that do not necessarily fall off with increase in the distance and so the typical properties of entangled wave functions. Thus, even though the wave function spreads out, the effects of the quantum potential need not necessarily decrease (as the equation of motion (10) of the many-body systems shows clearly, the total force acting on the $i$-th particle coming from the quantum potential, i.e. $\nabla_{i} Q$, does not necessarily fall off with increase in the distance; indeed, the forces between two particles of a many-body system may become stronger, even if $|\psi|$ may decrease in this limit). This is just the type of behavior required to explain the EPR paradox.

If we examine the expression of the quantum potential in the double-slit experiment, we find that it depends on the width of the slits, their distance apart, and the momentum of the particle. This means that the quantum potential has a contextual nature, namely brings a global information on the process and its environment; and it has an active information in the sense that it modifies the behavior of the particle. In a double-slit experiment, if one of the two slits is closed, the quantum potential changes, and this information arrives instantaneously to the particle, which behaves as a consequence.

The fact that the quantum potential produces an active information, a global information on the environment means that it cannot be seen as an external entity in space but as an entity, which contains a spatial information, i.e. as an entity, which represents space. It is thus possible to interpret the quantum potential (2) (or the analogous one (8) for a many-body system) as a geometrodynamic entity. The quantum potential has indeed a geometric nature just because has a contextual nature, contains a global information on the environment, in which the experiment is performed; and, at the same time, is a dynamical entity just because its information about the process and the environment is active, by determining the behavior of the particle. In this geometrodynamic picture, we can say that the quantum potential indicates and represents the geometric properties of space, from which the quantum force and, thus, the behavior of quantum particles, are derived [15]. As for the double-slit experiment, the fact that quantum potential is linked to the width of the slits, their distance apart, and the momentum of the particle, by presenting a global information on the environment, means just that it describes the geometric properties of the experimental arrangement (and, therefore, of space), which determine the quantum force and the behavior of the particle. Moreover, as we have already underlined above, the presence of the Laplace operator indicates that the geometric properties contained in quantum potential determine a non-local instantaneous action onto the particle. We can say therefore that Bohm's theory manages to manifest this essential feature of quantum mechanics, just by means of the geometric properties of space described and expressed by the quantum potential. In virtue of the features of the quantum potential, namely in virtue of the geometric properties of space, which de- 
termine the quantum forces, Bohm's theory turns out to be intrinsically holistic, in which "the whole is more than the sum of the parts". It is a merit of the pilot wave theory (and, in particular, of the geometrodynamic nature of quantum potential) to show, in such direct way, the non-locality that, according to Bohm, "... is the newest and most fundamental ontological characteristic implied by quantum theory" [16].

Finally, it is important to underline that, in the context of non-relativistic Bohmian mechanics, the Bohmian path integral can be associated with a Bohmian trajectory of the particle under consideration (and which is determined by the combined action of the classical potential and the quantum potential). In this regard, in the paper "The path integral approach in the frame work of causal interpretation" Abolhasani and Golshani showed that the propagation of the wave function in the context of the de Broglie-Bohm theory for a one-body system, can be achieved by means of a Bohmian path integral, which is defined, for two points $(x ; t)$ and $\left(x_{0} ; t_{0}\right)$ with a finite distance on a Bohmian path, by the relation

$$
\begin{aligned}
& \psi(\mathbf{x}, t)=\exp \left\{\frac{i}{\hbar} \int_{\mathbf{x}_{0}, t_{0}}^{\mathbf{x}, t}\left[\frac{(\nabla S)^{2}}{2 m}-(Q+V)\right] d t-\right. \\
& \left.-\int_{\mathbf{x}_{0}, t_{0}}^{\mathbf{x}, t} \frac{\nabla^{2} S}{2 m} d t\right\} \psi\left(\mathbf{x}_{0}, t_{0}\right),
\end{aligned}
$$

where the first exponential function can be obtained by integrating the quantum Hamilton-Jacobi equation (1) on the Bohmian path, while the second exponential one can be obtained by integrating the continuity equation (6) [17]. Equation (12) shows that the classical action (described by the Feynman path integral) is replaced by the quantum action (which is linked with the quantum potential $Q$ given by Eq. (2)). The Bohmian path integral given by Eq. (12) can also be extended to the case of a system of $N$ particles in the following way:

$$
\psi\left(\mathbf{x}_{1}, \ldots, \mathbf{x}_{N}, t\right)=\exp \left\{\frac{i}{\hbar} \int_{\mathbf{x}_{0}, t_{0}}^{\mathbf{x}, t} \sum_{i=1}^{N}\left[\frac{\left(\nabla_{i} S\right)^{2}}{2 m_{i}}-(Q+V)\right] d t-\right.
$$$$
\left.-=\int_{\mathbf{x}_{0}, t_{0}}^{\mathbf{x}, t} \sum_{i=1}^{N} \frac{\nabla_{i}^{2} S}{2 m_{i}} d t\right\} \psi\left(\mathbf{x}_{01}, \ldots, \mathbf{x}_{0 N}, t_{0}\right)
$$

where the quantum potential $Q$ is given by Eq. (8). In particular, Abolhasani and Golshani have determined $\psi(\mathbf{x}, t)$ for a free-wave packet in one dimension in terms of its Fourier components $e^{i k x}$ (for which $\nabla^{2} S=0$ and $Q=0)$. In this case, the Bohmian path integral (12) becomes

$\psi(x, t)=\int d k e^{-i \frac{\hbar k^{2}}{2 m}\left(t-t_{0}\right)} \varphi(k) e^{i k x}$

(which can be called Fourier-Bohm path integral). Abolhasani's and Golshani's treatment shows furthermore that, for the free wave packet in one dimension, the Feynman path integral can be obtained directly from the Bohmian path integral (12) on the basis of an "heuristic argument" (as a consequence of the fact that, in this simple case, the quantum potential vanishes).

\section{Quantum Entropy and the "Entropic Version" of the Non-Relativistic de Broglie-Bohm Theory}

The de Broglie-Bohm theory can receive a new interesting and suggestive reading which is based on the idea that all the features of the quantum potential follow from a fundamental physical quantity that can be appropriately called "quantum entropy". We call this new way of reading the de Broglie-Bohm theory as the "entropic version" of the Bohmian mechanics or, more briefly, "entropic Bohmian mechanics".

The entropic version of the non-relativistic Bohmian mechanics starts by defining the logarithmic function

$S_{Q}=-\frac{1}{2} \ln \rho$,

where $\rho$ is the probability density (describing the spacetemporal distribution of an ensemble of particles, namely the density of particles in the element of volume $d^{3} x$ around a point $\mathbf{x}$ at the time $t$ ) associated with the wave function $\psi(\mathbf{x}, t)$ of an individual physical system. As we know, in the Bohmian mechanics, each individual physical system is described contemporarily by a wave function and an ensemble of particles (distinguished by their initial locations). Now, in the entropic version of the Bohmian mechanics, we assume that the space-temporal distribution of the ensemble of particles describing the individual physical system under consideration generates a modification of the background space characterized by the quantity given by Eq. (15). Equation (15) presents some analogy with the standard definition of entropy given by the Boltzmann law: it provides, indeed, the quantum counterpart of a Boltzmann-type law. Since it shows a relation with the wave function, the quantity given by Eq. (15) can be appropriately defined as 
"quantum entropy". The quantum entropy (15) can be interpreted as the physical entity that characterizes, in the quantum domain, the degree of order and chaos of the vacuum - a storage of virtual trajectories supplying the optimal ones for the particle movement - which supports the density $\rho$ describing the space-temporal distribution of the ensemble of particles associated with the wave function under consideration. In the recent article Bohmian split of the Schrödinger equation onto two equations describing evolution of real functions, Sbitnev [18] has shown that, by introducing quantity (15), the quantum potential can be expressed in the following convenient way:

$Q=-\frac{\hbar^{2}}{2 m}\left(\nabla S_{Q}\right)^{2}+\frac{\hbar^{2}}{2 m}\left(\nabla^{2} S_{Q}\right)$

In this way, by substituting Eq. (16) into Eq. (3), we get the following equation of motion for the corpuscle associated with the wave function $\psi(\mathbf{x}, t)$ :

$\frac{|\nabla S|^{2}}{2 m}-\frac{\hbar^{2}}{2 m}\left(\nabla S_{Q}\right)^{2}+V+\frac{\hbar^{2}}{2 m}\left(\nabla^{2} S_{Q}\right)=-\frac{\partial S}{\partial t}$.

Equation (17) provides the energy conservation law, in which the term $-\frac{\hbar^{2}}{2 m}\left(\nabla S_{Q}\right)^{2}$ can be interpreted as the quantum corrector of the kinetic energy $\frac{|\nabla S|^{2}}{2 m}$ of the particle, while the term $\frac{\hbar^{2}}{2 m}\left(\nabla^{2} S_{Q}\right)$ can be interpreted as the quantum corrector of the potential energy $V$. On the ground of Sbitnev's results, the following reading of the quantum potential and the energy conservation law in quantum mechanics becomes permissible. The quantum potential can be derived from the quantum entropy describing the degree of order and chaos of the background space (namely, the modification in the background space) produced by the density of the ensemble of particles associated with the wave function under consideration. On the basis of Eq. (17), we can say that the quantum entropy determines two quantum corrections of the energy of the physical system under consideration (of the kinetic energy and of the potential energy, respectively). Without these two quantum corrections (linked just with the quantum entropy), the total energy of the system would not be conserved.

Moreover, by substituting the quantum entropy given by Eq. (15) in the continuity equation (6), we obtain the entropy balance equation

$\frac{\partial S_{Q}}{\partial t}=-\left(\mathbf{v} \cdot \nabla S_{Q}\right)+\frac{1}{2} \nabla \cdot \mathbf{v}$

where $\mathbf{v}=\frac{\nabla S}{m}$ is the particle's speed. In Eq. (18), the second term on the right-hand side describes the rate of entropy flow due to the spatial divergence of the speed. This second term is nonzero in the regions, where the particle changes the direction of movement. Since - on the basis of Brillouin's results - a negative value of $S_{Q}$ is related to information [19], Eq. (18) can be interpreted as a law, which describes the balance of information flows. As a consequence, the quantum potential expressed by Eq. (16), namely as a sum of two quantum correctors linked with the quantum entropy, can be interpreted as an information channel into the behavior of the particle under consideration. Moreover, in virtue of the dependence of the quantum potential on the quantum entropy, it is just the quantum entropy that can be considered the fundamental element that determines the fact that the quantum potential acts as an information channel into the behavior of the particle under consideration. The nature of the quantum potential to act as an information channel into the behavior of quantum particles is defined just by the quantum entropy. In other words, one can see that, by introducing the quantum entropy given by Eq. (15), just this quantity describing the degree of order and chaos of the vacuum - a storage of virtual trajectories supplying the optimal ones the particle movement - and supporting the density $\rho$ (of the particles associated with the wave function under consideration) is the fundamental element that produces an active information for the behavior of the particles at a fundamental level. The geometrodynamic nature of the quantum potential - namely the fact that the quantum potential has a geometric nature (a contextual nature), contains a global information on the environment, in which the experiment is performed, and the fact that, at the same time, it is a dynamical entity, namely its information about the process and the environment is active - can thus receive a new suggestive interpretation. It is just the quantum entropy as the fundamental element that produces the geometrodynamic nature of the quantum potential. The quantum potential expresses the geometric properties of space, from which the quantum force and, thus, the behavior of quantum particles follow. The quantum entropy (15) by indicating the degree of order and chaos of the vacuum as the background space (namely, a modification in the background space) produced by the density $\rho$ of the ensemble of particles associated with the wave function under consideration, represents which really are these geometric properties. The non-local action of the quantum potential itself can be seen as a consequence of the quantum entropy in virtue of the presence of the Laplace operator of the quantum entropy. 
The extension of the entropic non-relativistic Bohmian mechanics to many-body systems is straightforward. If we consider a wave function $\psi=$ $R\left(\mathbf{x}_{1}, \ldots, \mathbf{x}_{N}, t\right) e^{i S\left(\mathbf{x}_{1}, \ldots, \mathbf{x}_{N}, t\right) / \hbar}$ defined on the configuration space $R^{3 N}$ of a system of $N$ particles, the quantum entropy (15) associated with the action of the wave $\psi$ determines a many-body quantum potential given by the expression

$Q=\sum_{i=1}^{N}\left[-\frac{\hbar^{2}}{2 m_{i}}\left(\nabla_{i} S_{Q}\right)^{2}+\frac{\hbar^{2}}{2 m_{i}}\left(\nabla_{i}^{2} S_{Q} \cdot\right)\right]$

The equation of motion of the system determined by the quantum entropy (15) is as follows:

$\sum_{i=1}^{N} \frac{\left|\nabla_{i} S\right|^{2}}{2 m_{i}}-\sum_{i=1}^{N} \frac{\hbar^{2}}{2 m_{i}}\left(\nabla_{i} S_{Q}\right)^{2}+$

$+V+\sum_{i=1}^{N} \frac{\hbar^{2}}{2 m_{i}}\left(\nabla_{i}^{2} S_{Q}\right)=-\frac{\partial S}{\partial t}$

The continuity equation for the probability density becomes

$\frac{\partial S_{Q}}{\partial t}=\sum_{i=1}^{N}\left[-\left(\mathbf{v}_{i} \cdot \nabla_{i} S_{Q}\right)+\frac{1}{2} \nabla_{i} \cdot \mathbf{v}_{i}\right]$.

In synthesis, in the reading of the Bohmian mechanics based on the introduction of the quantum entropy given by Eq. (15), one can say that the quantum entropy can be indeed interpreted as a sort of the intermediary entity between the space and the behavior of quantum particles, and thus between the action of the quantum potential and the behavior of quantum particles. The introduction of the quantum entropy given by Eq. (15) as the fundamental entity that determines the behavior of quantum particles leads to two equations of motion, the energy conservation law (Eq. (17) for one-body systems and Eq. (20) for many-body systems), and the entropy balance equation (Eq. (18) for one-body systems and Eq. (21) for many-body systems), which introduce a new suggestive way to interpret the Bohmian mechanics. As we know, in the usual interpretation of the Bohmian mechanics, the equations of motion are nonlinear in nature via the dependence of the quantum potential on the wave function given by Eq. (2) (different initial conditions yield, in fact, different quantum potentials). Instead, now, in the entropic version of the Bohmian mechanics here proposed, one assumes preliminarily that the density of particles $\rho$ associated with the wave function of the physical system under consideration determines a modification in the background space and, thus, a certain degree of order and chaos defined by the logarithmic function (15). Then we can say that, in this "non-linear" background, the equations of motion of the system given now by Eqs. (17) and (18) (or, by Eqs. (20) and (21) for many-body systems) are "linear". As was shown already by Bittner, the introduction of the quantum entropy (15) allows one to transform a nonlinear model into a linear one [20]. The replacement of a non-linear model in the wave function with a linear model in the quantum entropy can be indeed considered as a relevant merit of the entropic version of the nonrelativistic Bohmian mechanics. By handling with the quantum entropy $S_{Q}$, it is possible to obtain some linear model of the non-relativistic Bohmian mechanics.

Moreover, it is of interest to remark that, in the approach of the quantum entropy, the de Broglie-Bohm path integrals can receive a new suggestive re-reading. By taking the new definition (16) of the quantum potential into account, Abolhasani's and Golshani's Bohmian path integral can be rewritten in the following convenient form:

$\psi(\mathbf{x}, t)=\exp \left\{\frac{i}{\hbar} \int_{\mathbf{x}_{0}, t_{0}}^{\mathbf{x}, t}\left[\frac{(\nabla S)^{2}}{2 m}+\frac{\hbar^{2}}{2 m}\left(\nabla S_{Q}\right)^{2}\right] d t-\right.$

$\left.-\int_{\mathbf{x}_{0}, t_{0}}^{\mathbf{x}, t}\left(\frac{\nabla^{2} S}{2 m}+\frac{\hbar^{2} \nabla^{2} S_{Q}}{2 m}+V\right) d t\right\} \psi\left(\mathbf{x}_{0}, t_{0}\right)$.

Equation (22) indicates clearly that the quantum entropy is the central element associated with a Bohmian path by determining the appropriate corrective terms into the kinetic energy and the potential energy (and thus into the Lagrangian) of the particle under consideration. In other words, in the entropic picture of the nonrelativistic Bohmian mechanics, the following re-reading of the Bohmian path integral formalism becomes permissible: the Bohmian path integral (22) is derived by integration of the quantum Lagrangian determined by the kinetic energy, the potential energy, and the quantum entropy of the particle, namely the quantum entropy describing the degree of order and chaos of the vacuum supporting the probability density introduces a modification of the Lagrangian and, thus, determines a particular Bohmian trajectory associated with the Bohmian path integral (22). 
Another important merit of the entropic nonrelativistic Bohmian mechanics is to lead to a complexified state space as the fundamental background. Let us consider, for simplicity, a one-body system. As regards the new definition (16) of the quantum potential in terms of the quantum entropy, by means of an opportune unification of the quantum Hamilton-Jacobi equation (17) and the entropy balance equation (18), a complexified Hamilton-Jacobi equation containing the complex kinetic and potential terms can be obtained. In this new approach, the two quantum correction terms of the kinetic energy and the potential energy both depending on the quantum entropy emerge as the fundamental terms that modify the classical Feynman's path integral by expanding coordinates and momenta to the imaginary sector.

In this regard, by multiplying Eq. (18) by $-i \hbar$ and substituting this result into Eq. (17), we obtain

$$
\begin{aligned}
& \frac{|\nabla S|^{2}}{2 m}+i \hbar \frac{1}{m}\left(\nabla S \cdot \nabla S_{Q}\right)-\frac{\hbar^{2}}{2 m}\left(\nabla S_{Q}\right)^{2}+ \\
& +V-i \hbar \frac{1}{2}(\nabla \mathbf{v})+\frac{\hbar^{2}}{2 m}\left(\nabla^{2} S_{Q}\right)=-\frac{\partial S}{\partial t} .
\end{aligned}
$$

By defining $J=S+i \hbar S_{Q}$ as a complexified action, the first three terms in (23) can be rewritten as the gradient of the complexified action squared

$$
\frac{|\nabla S|^{2}}{2 m}+i \hbar \frac{1}{m}\left(\nabla S \cdot \nabla S_{Q}\right)-\frac{\hbar^{2}}{2 m}\left(\nabla S_{Q}\right)^{2}=\frac{1}{2 m}(\nabla J)^{2} .
$$

As regards the other three terms of (23), they could stem from the expansion of the potential energy extended in the complex space possessing by a small broadening into the imaginary sector in Taylor's series (as regards the complex extension, see, e.g., [21]):

$$
\begin{aligned}
& V(\mathbf{x}+i \varepsilon) \approx V(\mathbf{x})+i \hbar\left(\mathbf{n} \cdot\left(\frac{s}{2 m} \nabla V(\mathbf{x})\right)\right)- \\
& -\frac{\hbar^{2}}{2 m}\left(\frac{s^{2}}{2 m} \nabla^{2} V(\mathbf{x})\right)+\ldots,
\end{aligned}
$$

where $\varepsilon=\frac{\hbar}{2 m} s \mathbf{n}$ is a small vector having the dimension of length, and $s$ is the universal constant, the reverse velocity [22], $s=4 \pi \varepsilon_{0} \frac{\hbar}{e^{2}}=4.57 \times 10^{-7}[\mathrm{~s} / \mathrm{m}], e$ is the elementary charge carried by a single electron, and $\varepsilon_{0}$ is the vacuum permittivity. As regards the second term of Eq. (25), we have a force $-\nabla V(\mathbf{x})$ multiplied by a vector $l \mathbf{n}$ providing, thus, an elementary work performed by this force at the shifting by a length $l$ along $\mathbf{n}$. The force multiplied by the factor $l \mathbf{n}$ and divided by the mass $m$ is the rate of velocity's variation per unit length, i.e., it represents the velocity divergence. So, the second term of Eq. (25) can be rewritten in the form

$\frac{s}{2 m}(\mathbf{n} \cdot \nabla V(\mathbf{x}))=-\frac{1}{2}(\nabla \cdot \mathbf{v})$.

The term $\left(\frac{s^{2}}{2 m} \nabla^{2} V(\mathbf{x})\right)$ is comparable with $S_{Q}$, so it can be made the position

$-\left(\frac{s^{2}}{2 m} \nabla^{2} V(\mathbf{x})\right)=\nabla^{2} S_{Q}$

Now, by defining the complexified momentum $\mathbf{p}^{\prime}=$ $\nabla J=\nabla S+i \hbar \nabla S_{Q}$ and the complexified coordinates $\mathbf{x}^{\prime}=\mathbf{x}+i \varepsilon$, Eq. (23) can be rewritten as a complexified Hamilton-Jacobi equation

$-\frac{\partial J}{\partial t}=\frac{1}{2 m}(\nabla J)^{2}+V\left(\mathbf{x}^{\prime}\right)=H\left(\mathbf{x}^{\prime}, \mathbf{p}^{\prime}, t\right)$,

where $H\left(\mathbf{x}^{\prime}, \mathbf{p}^{\prime}, t\right)$ on the right-hand side is the complexified Hamiltonian. The total derivative of the complex action gives the equation

$\frac{d J}{d t}=-H\left(\mathbf{x}^{\prime}, \mathbf{p}^{\prime}, t\right)+\sum_{i=1}^{N} p_{i}{ }^{\prime} \dot{x}_{i}^{\prime}=L\left(\mathbf{x}^{\prime}, \dot{\mathbf{x}}^{\prime}, t\right)$.

By integrating Eqs. (28) and (29), we obtain the solutions

$J=-\int_{t_{0}}^{t} H\left(\mathbf{x}^{\prime}, \mathbf{p}^{\prime}, \tau\right) d \tau+C_{1}$

$J=-\int_{t_{0}}^{t} L\left(\mathbf{x}^{\prime}, \dot{\mathbf{x}}^{\prime}, \tau\right) d \tau+C_{2}$

where $C_{1}$ and $C_{2}$ are two integration constants that satisfy the condition

$C_{1}-C_{2}=\int_{t_{0}}^{t} \sum_{i=1}^{N} p_{i}^{\prime} \dot{x}_{i}^{\prime} d t=\int_{L} \sum_{i=1}^{N} p_{i}^{\prime} d x_{i}^{\prime}$.

In Eq. (32), $L$ is a curve beginning at $t_{0}$ and terminating at $t$.

As it has been shown clearly by Sbitnev in his recent work "Bohmian trajectories and the Path Integral Paradigm. Complexified Lagrangian Mechanics", the 
complexified state space described by Eqs. (28), (29), (30), (31), and (32) (and therefore the quantum entropy) can be considered as the fundamental stage, which determines the features of Bohmian trajectories: the Bohmian trajectories are trajectories submitted to the principle of least action that expands on the action integral (31) containing the complexified Lagrangian function derived from the quantum entropy [23]. The Bohmian trajectories turn out to be the geodesic trajectories of an incompressible fluid loaded by the complexified Lagrangian that is determined, in turn, by the quantum potential expressed by Eq. (16), in other words, by the two quantum corrections determined by the quantum entropy.

In the complexified state space defined by Eqs. (28)(31) and (32), a solution of the Schrödinger equation can be written as

$\psi\left(\mathbf{x}^{\prime}, \mathbf{p}^{\prime}, t\right)=\exp \left(\frac{i}{\hbar} J\right)=\exp \left(\frac{i}{\hbar} S-S_{Q}\right)$.

By substituting the action integral (30) into Eq. (33), we obtain

$\psi\left(\mathbf{x}^{\prime}, \mathbf{p}^{\prime}, t\right)=\frac{1}{Z_{1}} \exp \left\{-\frac{i}{\hbar} \int_{t_{0}}^{t} H\left(\mathbf{x}^{\prime}, \mathbf{p}^{\prime}, \tau\right) d \tau\right\}$,

where $Z_{1}=\exp \left(-\frac{i}{\hbar} C_{1}\right)$. The probability density becomes

$\rho=\exp \left(-2 S_{Q}\right)$

In this complexified state space, Hamilton's principle $\delta J=0$ states that the motion of an arbitrary mechanical system occurs in such a way that the definite integral (31) becomes stationary for arbitrary possible variations of the configuration of the system, provided the initial and final configurations of the system are prescribed. This principle can also be reformulated with respect to the wave function expressed in terms of the complexified action (31)

$\psi\left(\mathbf{x}^{\prime}, \mathbf{p}^{\prime}, t\right)=\frac{1}{Z_{2}} \exp \left\{-\frac{i}{\hbar} \int_{t_{0}}^{t} L\left(\mathbf{x}^{\prime}, \dot{\mathbf{x}}^{\prime}, \tau\right) d \tau\right\}$,

where $Z_{2}=\exp \left(-\frac{i}{\hbar} C_{2}\right)$. In this case, the principle states: this exponent becomes stationary for arbitrary possible variations of the configuration of the system, provided the initial and final configurations of the system are prescribed. Obviously, this results from the stationarity of integral (31) stated above.
Moreover, the two Bohmian quantum corrections determined by the quantum entropy emerge as indispensable terms that modify the Feynman's path integral by expanding the coordinates and the momenta to the imaginary sector. As shown by Grosche [24], Feynman's path integral can be written mathematically in the following way in the complexified state space:

$K\left(\mathbf{x}^{\prime}, t ; \mathbf{x}_{0}^{\prime}, t_{0}\right)=$

$=\iint \ldots \int D\left[\mathbf{x}^{\prime}(\tau)\right] \exp \left\{\frac{i}{\hbar} \int_{t_{0}}^{t} L\left(\mathbf{x}^{\prime}, \dot{\mathbf{x}}^{\prime}, \tau\right) d \tau\right\}$.

Here, the path-integral symbol indicates the multiple integral

$\iint \ldots \int D\left[\mathbf{x}^{\prime}(\tau)\right] \Leftrightarrow$

$\Leftrightarrow\left(\frac{2 \pi i \hbar \delta t}{m}\right)^{-M / 2} \int_{\mathbf{x}_{o}^{\prime}}^{\mathbf{x}^{\prime}} d \mathbf{x}_{1}^{\prime} \int_{\mathbf{x}_{o}^{\prime}}^{\mathbf{x}^{\prime}} d \mathbf{x}_{2}^{\prime} \cdots \int_{\mathbf{x}_{o}^{\prime}}^{\mathbf{x}^{\prime}} d \mathbf{x}_{M}^{\prime}$.

The fundamental principle of quantum mechanics, namely the principle of superposition, underlies the path integral (37). Whereas the evolution of a classical object is described by a unique trajectory satisfying the principle of least action, the path integral tests all possible virtual classical trajectories, among which there is the unique trajectory satisfying the least action principle. Other trajectories cancel each other by their interference.

On the basis of Sbitnev's results [23], the interpretation of Feynman's path integral approach based on Eqs. (37) and (38) seems simple and natural in the complexified state space characterized by the complexified momenta $\mathbf{p}^{\prime}=\nabla J=\nabla S+i \hbar \nabla S_{Q}$ and by the complexified coordinates $\mathbf{x}^{\prime}=\mathbf{x}+i \varepsilon$ : the key of reading is provided by the two Bohmian quantum correctors linked with the quantum entropy. The path integral computation stems directly from the decomposition of the Schrödinger equation into the modified quantum Hamilton-Jacobi equation plus the entropy balance equation. The two Bohmian quantum correctors linked with the quantum entropy (15) and resulted from this decomposition allow the expanding of the state space to the imaginary sector. The imaginary terms emergent in this computations suppress the wilder contributions to the path integral. Thus, we have a non-trivial $N$-dimensional manifold embedded in the $2 N$-dimensional complex state space, where its real part is the conventional coordinate state space. 


\section{Entropic Version of the Relativistic de Broglie-Bohm Theory in a Curved Space-Time}

The next important step is to develop an entropic version of the relativistic de Broglie-Bohm theory in a curved space-time and to show the perspectives introduced by the quantum entropy in this field. In this regard, we will consider the interesting Bohmian approach to the KleinGordon equation developed by F. Shojai and A. Shojai in [25], [26]. In this model, the quantum Hamilton-Jacobi equation which can be derived by the decomposition of the wave function in its polar form $\psi=|\psi| \exp \left(\frac{i S}{\hbar}\right)$ has the form

$\partial_{\mu} S \partial^{\mu} S=m^{2} c^{2} \exp Q$

which is Poincarè-invariant and has the correct nonrelativistic limit. Here, the quantum potential is defined as

$Q=\frac{\hbar^{2}}{m^{2} c^{2}} \frac{\left(\nabla^{2}-\frac{1}{c^{2}} \frac{\partial^{2}}{\partial t^{2}}\right)|\psi|}{|\psi|}$.

The continuity equation is

$\partial_{\mu}\left(\rho \partial^{\mu} S\right)=0$

where $\rho$ is the density of particles in the element of volume $d^{3} x$ around a point $\mathbf{x}$ at the time $t$ associated with the wave function $\psi(\mathbf{x}, t)$ of the individual physical system under consideration. Just like in the non-relativistic problem, we start by defining the quantum entropy

$S_{Q}=-\frac{1}{2} \ln \rho$.

In the entropic version of the Bohmian relativistic quantum mechanics, the space-temporal distribution of the ensemble of particles describing the individual physical system under consideration is assumed to generate a modification and, thus, a degree of order and chaos of the background space characterized by the quantity given by Eq. (42). This modification of the background space described by the quantum entropy (42) determines a quantum potential given by the relation

$Q=-\frac{\hbar^{2}}{m^{2} c^{2}}\left(\partial_{\mu} S_{Q}\right)^{2}+\frac{\hbar^{2}}{m^{2} c^{2}}\left(\left(\nabla^{2}-\frac{1}{c^{2}} \frac{\partial^{2}}{\partial t^{2}}\right) S_{Q}\right)$.

So, the quantum Hamilton-Jacobi equation (39) becomes

$\partial_{\mu} S \partial^{\mu} S=m^{2} c^{2} \exp \left[-\frac{\hbar^{2}}{m^{2} c^{2}}\left(\partial_{\mu} S_{Q}\right)^{2}+\right.$ $\left.+\frac{\hbar^{2}}{m^{2} c^{2}}\left(\left(\nabla^{2}-\frac{1}{c^{2}} \frac{\partial^{2}}{\partial t^{2}}\right) S_{Q}\right)\right]$

while the continuity equation (41) becomes

$\frac{1}{c} \frac{\partial S_{Q}}{\partial t}=-\left(p^{\mu} \partial_{\mu} S_{Q}\right)+\frac{1}{2} \partial_{\mu} p^{\mu}$.

Equation (44) and Eq. (45) can be considered as the fundamental equations of motion in the entropic view of the Bohmian Klein-Gordon relativistic quantum mechanics. Since, on the basis of the relativistic quantum Hamilton-Jacobi equation (39), the quantum potential turns out to be essentially the mass square, and since Eq. (39) emerges in the entropic picture as a direct consequence of the more fundamental equation (44) (because the quantum potential is determined by the quantum entropy), we may say within our approach that the mass square in Eq. (39) is determined by the quantum entropy. Therefore, the very interesting perspective emerges that, in the entropic approach to the relativistic de Broglie-Bohm theory, the quantum mass defined by the relation

$M^{2}=m^{2} \exp Q$

is generated by the quantum entropy, by the degree of order and chaos of the vacuum, and by the modification of the background space caused by the density of particles associated with the wave function under consideration.

Moreover, F. Shojai and A. Shojai [26] have shown that, as regards Bohm's version of the Klein-Gordon equation, by changing the ordinary differentiation $\partial_{\mu}$ with the covariant derivative $\nabla_{\mu}$ and by changing the Lorentz metric with the curved metric $g_{\mu \nu}$ in Eqs. (39), (40), and (41), it is possible to combine the de BroglieBohm quantum theory of motion and the theory of gravity and to interpret the quantum potential as the conformal degree of freedom of the space-time metric. In other words, in F. Shojai's and A. Shojai's model, the effects of the gravity on the geometry and the quantum effects on the geometry of space-time are highly coupled. The geometric properties which are expressed by the quantum potential and determine the behavior of a zero-spin particle are linked with the curved space-time: the particles determine the curvature of space-time, and, at the same time, the space-time metric is linked with the quantum potential, which influences the behavior of the particles. Now, by starting from the quantum entropy (42), an entropic version of the relativistic de Broglie-Bohm theory in a curved space-time can be suggested, in which it is possible to explain why and in what sense the quantum potential is the conformal degree of freedom of the 
space-time metric, why and in what sense the effects of gravity on the geometry and the quantum effects on the geometry of space-time are highly coupled: the key to the explanation of these results lies just in the quantum entropy, in the modification of the background space determined by the density of particles associated with the wave function under consideration.

In the entropic approach, the equations of motion for a particle (of spin 0) in a curved background take the form

$$
\frac{1}{c} \frac{\partial S_{Q}}{\partial t}=-\left(p_{\mu} \nabla^{\mu} S_{Q}\right)+\frac{1}{2} \nabla_{\mu} p^{\mu}
$$

$g^{\mu \nu} \nabla_{\mu} S \nabla^{\mu} S=m^{2} c^{2} \exp \left[-\frac{\hbar^{2}}{m^{2} c^{2}}\left(\nabla_{\mu} S_{Q}\right)^{2}+\right.$

$\left.+\frac{\hbar^{2}}{m^{2} c^{2}}\left(\left(\nabla^{2}-\frac{1}{c^{2}} \frac{\partial^{2}}{\partial t^{2}}\right)_{g} S_{Q},\right)\right]$

and the quantum potential is

$$
Q=-\frac{\hbar^{2}}{m^{2} c^{2}}\left(\nabla_{\mu} S_{Q}\right)^{2}+\frac{\hbar^{2}}{m^{2} c^{2}}\left(\left(\nabla^{2}-\frac{1}{c^{2}} \frac{\partial^{2}}{\partial t^{2}}\right)_{g} S_{Q}\right)
$$

Utilizing now the fruitful de Broglie's observation [27] that the quantum theory of motion for relativistic spinless particles is very similar to the classical theory of motion in a conformally flat space-time, in which the conformal factor is related to Bohm's quantum potential, the quantum Hamilton-Jacobi equation (48) can be written equivalently as

$\frac{m^{2}}{M^{2}} g^{\mu \nu} \nabla_{\mu} S \nabla_{\nu} S=m^{2} c^{2}$

where

$$
\begin{aligned}
& M^{2}=m^{2} \exp \left[-\frac{\hbar^{2}}{m^{2} c^{2}}\left(\nabla_{\mu} S_{Q}\right)^{2}+\right. \\
& \left.+\frac{\hbar^{2}}{m^{2} c^{2}}\left(\left(\nabla^{2}-\frac{1}{c^{2}} \frac{\partial^{2}}{\partial t^{2}}\right)_{g} S_{Q}\right)\right] .
\end{aligned}
$$

From this relation, it can be concluded that the quantum effects are equivalent to a change of the space-time metric from $g_{\mu \nu}$ to

$\tilde{g}_{\mu \nu}=\frac{M^{2}}{m^{2}} g_{\mu \nu}$, which is a conformal transformation, which is determined, indeed, by the quantum entropy (42) and, thus, by the degree of order and chaos of the vacuum, by a modification of the background space caused by the density of particles associated with the wave function under consideration. In this way, Eq. (50) can be written as

$\tilde{g}^{\mu \nu} \tilde{\nabla}_{\mu} S \tilde{\nabla}_{\nu} S=m^{2} c^{2}$,

where $\tilde{\nabla}_{\mu}$ represents the covariant differentiation with respect to the metric $\tilde{g}_{\mu \nu}$. Moreover, in this new curved space-time, the continuity equation takes the form

$\tilde{g}^{\mu \nu} \frac{1}{c} \frac{\partial S_{Q}}{\partial t}=\tilde{g}^{\mu \nu}\left[-\left(p_{\mu} \tilde{\nabla}^{\mu} S_{Q}\right)+\frac{1}{2} \nabla_{\mu} p^{\mu}\right]$.

The important conclusion we can draw from this treatment is that the presence of the quantum potential is equivalent to a curved space-time with its metric being given by (52), where the mass square is just determined by the quantum entropy. In this way, we have achieved a geometrization of the quantum aspects of matter in a picture based on the idea that the density of particles associated with a given wave function determines a modification of the background space.

It seems that there is a dual aspect to the role of geometry in physics. The space-time geometry sometimes looks like what we call gravity and sometimes looks like what we understand as quantum behavior. The real key to the reading of this link lies just in the quantum entropy: the effects of gravity on geometry and the quantum effects on the geometry of space-time are highly coupled, because they are both determined by the background space described by the quantum entropy, and they are both produced by the degree of order and chaos of the vacuum supporting the density of particles associated with the wave function under consideration. The entropic treatment of the motion of a zero-spin particle in a curved background introduces, therefore, the interesting perspectives: in fact, on the ground of relations (50) and (51), we can say that the geometric properties, which are expressed by the quantum entropy and which determine the behavior of a zero-spin particle are linked to the curved space-time. In other words, we can say that the particles determine the curvature of space-time, and, at the same time, the space-time metric is linked with the quantum entropy, which influences the behavior of the particles. The quantum entropy appears, indeed, as a real intermediary between gravitational and quantum effects of matter. Quantum entropy itself generates a curvature, which may have a large influence on the classical contribution to the curvature of the space-time. 
It can be also interesting to observe that the particle trajectory can be derived from the guidance formula and by differentiating Eq. (48) with the use of the above conformal transformation, which leads to a Newton-type equation of motion

$M \frac{d^{2} x^{\mu}}{d \tau^{2}}+M \Gamma_{\nu \kappa}^{\mu} u^{\nu} u^{\kappa}=\left(c^{2} g^{\mu \nu}-u^{\mu} u^{\nu}\right) \nabla_{\nu} M$

Equation (55) reduces to the standard geodesic equation via the above conformal transformation (52). By taking Eq. (51) into account, the quantum entropy can be considered as the fundamental element, which determines the particle trajectory described by the Newtontype equation of motion (55).

\section{Perspectives of the Quantum Entropy in the Bohmian Quantum Gravity}

In this section, we will introduce an entropic version of the de Broglie-Bohm theory in the quantum gravity domain. In particular in the first part, we will show the perspectives introduced by the quantum entropy in the context of the scalar-tensor Bohmian model regarding quantum gravity. In the second part, we will present some considerations about the Wheeler-DeWitt equation.

Before all, we remember that, despite some problems and weak points (for example, the fact that it is still an open question among the so-called Bohmian community which sense to give - if any - to the wave function of the Universe), if we consider some recent research, the Bohmian interpretation of canonical quantum gravity turns out to have several useful aspects and merits [7, 28-31].

Some of them are the following ones:

- It leads to the time evolution of the dynamical variables whether the wave function depends on the time or not. Therefore, in the Bohmian quantum gravity, we have no time problem.

- Bohm's theory describes a single system, unlike the standard interpretation of quantum theory, which does not tell anything about a single system. About an ensemble of the system, both interpretations are equivalent. This is because of the specific form of Bohm's equations of motion. They are the Bohmian version of the Hamilton-Jacobi equation and the conservation equation of probability density. These equations can be transformed to the
Schrödinger equation by some canonical transformation. This aspect is useful in quantum cosmology, where the system is the Universe, and an ensemble of systems does not exist. Therefore, from the Bohmian point of view, we have no conceptual problem of the meaning of Universe's wave function in quantum cosmology.

- Normalization of the wave function is needed only for the probabilistic description. Here, there is no need to normalize the wave function for a single system.

- The classical limit has a well-defined meaning. When the quantum potential is less than the classical potential, and the quantum force is less than the classical force, we are in the classical domain.

- There is no need to separate the classical observer and the quantum system in the measurement problem. In the Bohmian picture of the measurement process, we have two interacting systems, the system and the observer. After the interaction takes place, the wave function of the system is reduced in a causal way. It must be noted that the same statistical results for the standard and Bohmian interpretations do not mean that the two theories are equivalent. They are different in physical concepts. The most important difference is that one deals in the Bohmian interpretation with trajectories. This can lead to new concepts. For example, one can evaluate the tunneling time of a particle through the potential barrier in the non-relativistic quantum mechanics. This is a concept that has no clear meaning in the standard interpretation [7, 32].

Till now, the Bohmian interpretation of the Wheeler-De Witt quantum gravity and cosmology has given some physical results that can be found in the literature:

- In the Bohmian quantum cosmology, the quantum force can remove the Big-Bang singularity, because it can behave as a repulsive force $[33,34]$.

- The quantum force may be present on large scales, because the quantum effects of the quantum potential are independent of the scale [35].

- One can find the graceful exit behavior in the superinflation model in a super string cosmology. The evolution begins with the inflation and smoothly changes to the decelerating expansion, without any singularity in the transition [36]. For 
a more detailed discussion of the de Broglie-Bohm interpretation of quantum super string cosmology, the pre-Big-Bang inflation, and the graceful exit problem considering various classes of wavepackets, see [37].

- Real time tunneling can occur in the classically forbidden regions through the quantum potential. For this effect in a closed de Sitter Universe in $2+1$ dimensions, see [38].

- Finally, in a generalized geometric picture of Bohm's interpretation, one can unify the quantum effects and gravity [34, 39-44].

The latest point represents a very important result that the Bohmian version of quantum gravity can achieve. In this regard, F. Shojai and A. Shojai developed a toy model of quantum gravity (providing a scalar-tensor picture of the model mentioned in Section 4 ), in which the form of the quantum potential and its relation to the conformal degree of freedom of the spacetime metric can be derived, by using the equations of motion. By showing that it is just the quantum gravity equations of motion that make the quantum potential the entity expressing the geometric properties, which influences the behavior of the particles and which is related to the space-time metric, F. Shojai's and A. Shojai's model suggests a sort of unification of the gravitational and quantum aspects of matter at a fundamental level of physical reality.

Here, we want to develop an entropic version of $\mathrm{F}$. Shojai's and A. Shojai's toy model of quantum gravity. This approach can be considered a relevant and interesting development in the interpretation of the quantum entropy, as the ultimate visiting card, also in the quantum gravity domain.

A general relativistic system consisting of gravity and classical matter can be determined by the action

$$
\begin{aligned}
& A_{\text {no-quantum }}=\frac{1}{2 k} \int d^{4} x \sqrt{-g} R+ \\
& +\int d^{4} x \sqrt{-g} \frac{\hbar^{2}}{m}\left(\frac{\rho}{\hbar^{2}} \partial_{\mu} S \partial^{\mu} S-\frac{m^{2}}{\hbar^{2}} \rho\right),
\end{aligned}
$$

where $\rho=J^{0}$ is the ensemble density of the particles, $k=8 \pi G$, and hereafter we chose the units, in which $c=1$. On the other hand, as we have mentioned in Section 4, the introduction of quantum effects is equivalent to a change of the space-time metric from $g_{\mu \nu}$ to $g_{\mu \nu} \rightarrow g_{\mu \nu}^{I}=\frac{g_{\mu \nu}}{\exp Q}$, which is a conformal transformation (that, as we have shown in Section 4, is determined by the quantum entropy). Therefore, in order to introduce quantum effects, we make this conformal transformation, instead of adding the quantum potential term [25].

In this regard, we can write the action with quantum effects as

$A\left[\bar{g}_{\mu \nu}, \Omega, S, \rho, \lambda\right]=\frac{1}{2 k} \int d^{4} x \sqrt{-\bar{g}}\left(\bar{R} \Omega^{2}-6 \bar{\nabla}_{\mu} \Omega \bar{\nabla}^{\mu} \Omega\right)+$

$+\int d^{4} x \sqrt{-\bar{g}}\left(\frac{\rho}{m} \Omega^{2} \bar{\nabla}_{\mu} S \bar{\nabla}^{\mu} S-m \rho \Omega^{4}\right)+$

$+\int d^{4} x \sqrt{-\bar{g}} \lambda\left(\Omega^{2}-\left(1+\frac{\hbar^{2}\left(\nabla^{2}-\frac{\partial^{2}}{\partial t^{2}}\right) \sqrt{\rho}}{m^{2} \sqrt{\rho}}\right)\right)$

where $\Omega^{2}=\exp Q$ is the conformal factor, a bar over any quantity means that it corresponds to the no-quantum regime, and $\lambda$ is a Lagrange multiplier introduced in order to identify the conformal factor with its Bohmian value.

By the variation of the above action with respect to $\bar{g}_{\mu \nu}, \Omega, \rho, S$, and $\lambda$, we arrive at the following relations as our equations of motion:

1. The equation of motion for $\Omega$ :

$\bar{R} \Omega+6\left(\bar{\nabla}^{2}-\frac{\bar{\partial}^{2}}{\partial t^{2}}\right) \Omega+$

$+2 \frac{k}{m} \rho \Omega\left(\bar{\nabla}_{\mu} S \bar{\nabla}^{\mu} S-2 m^{2} \Omega^{2}\right)+2 k \lambda \Omega=0 ;$

2. The continuity equation for the particles:

$\bar{\nabla}_{\mu}\left(\rho \Omega^{2} \bar{\nabla}^{\mu} S\right)=0$

3. The equation of motion for the particles:

$\left(\bar{\nabla}_{\mu} S \bar{\nabla}^{\mu} S-m^{2} \Omega^{2}\right) \Omega^{2} \sqrt{\rho}+\frac{\hbar^{2}}{2 m} \times$

$\times\left[\left(\bar{\nabla}^{2}-\frac{\bar{\partial}^{2}}{\partial t^{2}}\right)\left(\frac{\lambda}{\sqrt{\rho}}\right)-\lambda \frac{\left(\bar{\nabla}^{2}-\frac{\bar{\partial}^{2}}{\partial t^{2}}\right) \sqrt{\rho}}{\rho}\right]=0$ 
4. The modified Einstein equations for $\bar{g}_{\mu \nu}$ :

$$
\Omega^{2}\left[\bar{R}_{\mu \nu}-\frac{1}{2} \bar{g}_{\mu \nu} \bar{R}\right]-\left[\bar{g}_{\mu \nu}\left(\bar{\nabla}^{2}-\frac{\bar{\partial}^{2}}{\partial t^{2}}\right)-\bar{\nabla}_{\mu} \bar{\nabla}_{\nu}\right] \Omega^{2}-
$$$$
-6 \bar{\nabla}_{\mu} \Omega \bar{\nabla}_{\nu} \Omega+3 \bar{g}_{\mu \nu} \bar{\nabla}_{\alpha} \Omega \bar{\nabla}^{\alpha} \Omega+\frac{2 k}{m} \rho \Omega^{2} \bar{\nabla}_{\mu} S \bar{\nabla}_{\nu} S-
$$$$
-\frac{k}{m} \rho \Omega^{2} \bar{g}_{\mu \nu} \bar{\nabla}_{\alpha} S \bar{\nabla}^{\alpha} S+k m \rho \Omega^{4} \bar{g}_{\mu \nu}+\frac{k \hbar^{2}}{m^{2}} \times
$$$$
\times\left[\bar{\nabla}_{\mu} \sqrt{\rho} \bar{\nabla}_{\nu}\left(\frac{\lambda}{\sqrt{\rho}}\right)+\bar{\nabla}_{\nu} \sqrt{\rho} \bar{\nabla}_{\mu}\left(\frac{\lambda}{\sqrt{\rho}}\right)\right]-
$$$$
-\frac{k \hbar^{2}}{m^{2}} \bar{g}_{\mu \nu} \bar{\nabla}_{\alpha}\left[\lambda \frac{\bar{\nabla}^{\alpha} \sqrt{\rho}}{\sqrt{\rho}}\right]=0
$$

5. The constraint equation:

$$
\Omega^{2}=1+\frac{\hbar^{2}}{m^{2}} \frac{\left(\bar{\nabla}^{2}-\frac{\bar{\partial}^{2}}{\partial t^{2}}\right) \sqrt{\rho}}{\sqrt{\rho}} .
$$

Now in the entropic picture, the conformal factor $\Omega^{2}=$ $\exp Q$ is determined by the background space described by the quantum entropy on the basis of the relation

$$
\begin{aligned}
& \Omega^{2}=\exp \left[-\frac{\hbar^{2}}{m^{2} c^{2}}\left(\nabla_{\mu} S_{Q}\right)^{2}+\right. \\
& \left.+\frac{\hbar^{2}}{m^{2} c^{2}}\left(\left(\nabla^{2}-\frac{1}{c^{2}} \frac{\partial^{2}}{\partial t^{2}}\right)_{g} S_{Q}\right)\right]
\end{aligned}
$$

and, thus, is produced by the degree of order and chaos of the vacuum supporting the density of particles associated with the wave function under consideration. By taking Eq. (63) into account, the constraint equation (62) can be also expressed in the equivalent form

$\exp \left[-\frac{\hbar^{2}}{m^{2} c^{2}}\left(\nabla_{\mu} S_{Q}\right)^{2}+\frac{\hbar^{2}}{m^{2} c^{2}}\left(\left(\nabla^{2}-\frac{1}{c^{2}} \frac{\partial^{2}}{\partial t^{2}}\right)_{g} S_{Q}\right)\right]=$

$$
=\frac{\hbar^{2}}{m^{2}} \frac{\left(\bar{\nabla}^{2}-\frac{\bar{\partial}^{2}}{\partial t^{2}}\right) \sqrt{\rho}}{\sqrt{\rho}}
$$

which shows the direct link between the density of particles and the quantum entropy in the quantum gravity domain. The equations of motion (58)-(62), in virtue of the link between the quantum potential with the quantum entropy, tell us that there are the back-reaction effects of the quantum factor on the background, which are due to the quantum entropy. On the basis of the highly coupled five equations above listed, one can say that, in the quantum gravity domain, the quantum entropy is the fundamental entity, the ultimate visiting card, which introduces the links (and, thus, the back-reaction terms) between the quantum effects and the background.

Moreover, in F. Shojai's and A. Shojai's model, by combining Eqs. (58) and (59), it is possible to arrive at a more simple relation instead of (58). If we use the trace of (61) and use (62), we have, after some mathematical manipulations,

$$
\lambda=\frac{\hbar^{2}}{m^{2}} \bar{\nabla}_{\mu}\left[\lambda \frac{\bar{\nabla}^{\mu} \sqrt{\rho}}{\sqrt{\rho}}\right]
$$

If one resolves this equation in perturbative way in terms of the parameter $\alpha=\frac{\hbar^{2}}{m^{2}}$ by writing $\lambda=\lambda^{(0)}+\alpha \lambda^{(1)}+$ $\alpha^{2} \lambda^{(2)}+\ldots$ and $\sqrt{\rho}=\sqrt{\rho}^{(0)}+\alpha \sqrt{\rho}^{(1)}+\alpha^{2} \sqrt{\rho}^{(2)}+\ldots$, one obtains

$\lambda^{(0)}=\lambda^{(1)}=\lambda^{(2)}=\ldots=0$.

Thus, the perturbative solution of

$\lambda=\frac{\hbar^{2}}{m^{2}} \bar{\nabla}_{\mu}\left[\lambda \frac{\bar{\nabla}^{\mu} \sqrt{\rho}}{\sqrt{\rho}}\right]$

is $\lambda=0$, which is its trivial solution. In this way, the equations of quantum gravity become

$\bar{\nabla}_{\mu}\left(\rho \Omega^{2} \bar{\nabla}^{\mu} S\right)=0$,

$\bar{\nabla}_{\mu} S \bar{\nabla}^{\mu} S=m^{2} \Omega^{2}$

$G_{\mu \nu}=-k T_{\mu \nu}^{(m)}-k T_{\mu \nu}^{(\Omega)}$,

where $T_{\mu \nu}^{(m)}$ is the matter energy-momentum tensor,

$k T_{\mu \nu}^{(\Omega)}=\frac{\left[g_{\mu \nu}\left(\nabla^{2}-\frac{\partial^{2}}{\partial t^{2}}\right)-\nabla_{\mu} \nabla_{\nu}\right] \Omega^{2}}{\Omega^{2}}+$

$+6 \frac{\nabla_{\mu} \Omega \nabla_{\nu} \Omega}{\omega^{2}}-3 g_{\mu \nu} \frac{\nabla_{\alpha} \Omega \nabla^{\alpha} \Omega}{\Omega^{2}}$,

and

$\Omega^{2}=1+\alpha \frac{\overline{\left(\nabla^{2}-\frac{\partial^{2}}{\partial t^{2}}\right)} \sqrt{\rho}}{\sqrt{\rho}}$. 
It can be noted that (69) is a Bohmian-type equation of motion. If we write it in terms of the physical metric $g_{\mu \nu}$, it reads

$\nabla_{\mu} S \nabla^{\mu} S=m^{2} c^{2}$

In all equations (68), (69), and (70) of quantum gravity, the quantum entropy turns out to be the ultimate visiting card, in virtue of the link between the conformal factor and the quantum entropy.

The next step is to make the conformal factor and the quantum potential (and, thus, the quantum entropy) dynamical. In the original F. Shojai's and A. Shojai's toy model, by starting from the most general scalar-tensor action

$A=\int d^{4} x\left\{\phi R-\frac{\omega}{\phi} \nabla^{\mu} \phi \nabla_{\mu} \phi+2 \Lambda \phi+L_{m}\right\}$,

in which $\omega$ is a constant independent of the scalar field $\phi, \Lambda$ is the cosmological constant, and $L_{m}$ is the matter Lagrangian (which is assumed to be in the form

$L_{m}=\frac{\rho}{m} \phi^{a} \nabla^{\mu} S \nabla_{\mu} S-m \rho \phi^{b}-\Lambda(1+Q)^{d}$,

in which $a, b$, and $d$ are constants), using a perturbative expansion for the scalar field and the matter distribution density as

$\phi=\phi_{0}+\alpha \phi_{1}+\ldots$

$\sqrt{\rho}=\sqrt{\rho_{0}}+\alpha \sqrt{\rho_{1}}+\ldots$

(and imposing the opportune physical constraints in order to determine the parameters $a, b$, and $d$ ), F. Shojai and A. Shojai have found the following quantum gravity equations:

$\phi=1+Q-\frac{\alpha}{2}\left(\nabla^{2}-\frac{\partial^{2}}{\partial t^{2}}\right) Q$

$\nabla^{\mu} S \nabla_{\mu} S=m^{2} \phi-\frac{2 \Lambda m}{\rho}(1+Q)(Q-\tilde{Q})+$

$+\frac{\alpha \Lambda m}{\rho}\left[\left(\nabla^{2}-\frac{\partial^{2}}{\partial t^{2}}\right) Q-2 \nabla_{\mu} Q \frac{\nabla^{\mu} \sqrt{\rho}}{\sqrt{\rho}}\right]$

$\nabla_{\mu}\left(\rho \nabla^{\mu} S\right)=0$

$G^{\mu \nu}-\Lambda g^{\mu \nu}=-\frac{1}{\phi} T^{\mu \nu}-\frac{1}{\phi}\left[\nabla^{\mu} \nabla^{\nu}-g^{\mu \nu}\left(\nabla^{2}-\frac{\partial^{2}}{\partial t^{2}}\right)\right] \times$ $\times \phi+\frac{\omega}{\phi^{2}} \nabla^{\mu} \phi \nabla^{\nu} \phi-\frac{1}{2} \frac{\omega}{\phi^{2}} g^{\mu \nu} \nabla^{\alpha} \phi \nabla_{\alpha} \phi$,

where

$Q=-\frac{\hbar^{2}}{m^{2} c^{2}}\left(\nabla_{\mu} S_{Q}\right)^{2}+\frac{\hbar^{2}}{m^{2} c^{2}}\left(\left(\nabla^{2}-\frac{1}{c^{2}} \frac{\partial^{2}}{\partial t^{2}}\right)_{g} S_{Q}\right)$

$\tilde{Q}=\alpha \frac{\nabla_{\mu} \sqrt{\rho} \nabla^{\mu} \sqrt{\rho}}{\sqrt{\rho}}$,

and

$T^{\mu \nu}=-\frac{1}{\sqrt{-g}} \frac{\delta}{\delta g_{\mu \nu}} \int d^{4} x \sqrt{-g} L_{m}$

is the energy-momentum tensor.

From the entropic viewpoint, this quantum gravity model suggested by F. Shojai and A. Shojai (and synthesized in Eqs. (76)-(79)) allows us to draw some important conclusions:

- In this model, Eq. (78) shows that the causal structure of the space-time $g^{\mu \nu}$ is determined by the gravitational effects of matter and, thus, by the quantum entropy, which must be considered as the ultimate entity, which shows that the quantum effects and the gravitational effects are coupled (also in the quantum gravity domain). On the basis of Eq. (76), quantum effects and, thus, the quantum entropy determine directly the scale factor of space-time.

- The mass field given by the right-hand side of Eq. (77) consists of two parts. The first part, which is proportional to $\alpha$, is a purely quantum effect, while the second part, which is proportional to $\alpha \Lambda$, is a mixture of the quantum effects and the large-scale structure introduced via the cosmological constant.

- In this model, the scalar field produces the quantum force, which appears on the right-hand side and violates the equivalence principle (just like in the Kaluza-Klein theory, the scalar field - dilaton - produces the fifth force leading to the violation of the equivalence principle [45]).

Finally, let us make some considerations about the perspectives introduced by the quantum entropy on the Wheeler-DeWitt (WDW) equation, which characterizes the wave-functional $\Psi$ of the Universe (here, we have made the position $\hbar=c=1$ ):

$\left[(8 \pi G) G_{a b c d} p^{a b} p^{c d}+\frac{1}{16 \pi G} \sqrt{g}\left(2 \Lambda-{ }^{(3)} R\right)\right] \Psi=0$. 
In the WDW equation (80), $G_{a b c d}=\frac{1}{2} \sqrt{g}\left(g_{a c} g_{b d}+\right.$ $\left.g_{a d} g_{b c}-g_{a b} g_{c d}\right)$ is the supermetric, $p^{a b}$ are the momentum operators related to the 3 -metric $g_{a b}, g=\operatorname{det} g_{i j}$, ${ }^{(3)} R$ is the 3 -dimensional curvature scalar, $\Lambda$ is the cosmological constant, and $G$ is the gravitational constant. The WDW equation (80) has the following important points:

1. The time parameter, which defines the foliation of the space-time, does not appear in it (we have, thus, the so-called time-problem in quantum gravity).

2. A different ordering of factors leads to different results.

3. In practice in order to solve the WDW equation, we must limit ourselves to a minisuperspace, in which some of the degrees of freedom are non-frozen, instead of using an infinite-dimensional superspace.

4. This is necessary for the wave-function to be square-integrable, in order to have a probabilistic interpretation for it. But it is not possible in all cases, because a precise definition of the inner product is not known in quantum gravity.

Here, we want to focus our attention on the Bohmian version of the WDW equation. In the Bohmian approach, by decomposing the wave functional $\Psi$ in the polar form $\Psi=R e^{i S / \hbar}$, one obtains the modified Hamilton-Jacobi equation

$(8 \pi G) G_{a b c d} \frac{\delta S}{\delta g_{a b}} \frac{\delta S}{\delta g_{c d}}-\frac{1}{16 \pi G} \sqrt{g}\left(2 \Lambda-{ }^{(3)} R\right)+Q_{G}=0$

where

$Q_{G}=\hbar^{2} N g G_{a b c d} \frac{1}{R} \frac{\delta^{2} R}{\delta g_{a b} \delta g_{c d}}$,

and $N$ being the lapse function. The term $Q_{G}$ can be defined as "quantum potential for the gravitational field". Equation (81) indicates that the only difference between classical and quantum Universes is the existence of the quantum potential in the latter. Moreover, it is important to mention that, in the Bohmian approach, Einstein's equations - in absence of source of matter-energy - take the form

$R^{\mu \nu}-\frac{1}{2} g^{\mu \nu} R=-\frac{1}{N} \frac{\delta \int Q_{G} d^{3} x}{\delta g_{i j}}$ for the dynamical parts and

$R^{0 \nu}-\frac{1}{2} g^{0 \nu} R=\frac{Q_{G}}{2 \sqrt{-g}} g^{0 \nu}$

for the non-dynamical part. (The reader can find some interesting developments as regards the Bohmian approach to the WDW equation, for example, in $[34,39$, 46-49].)

Let us define now a "quantum entropy for the gravitational field"

$S_{Q}=g \ln \rho$.

The quantum entropy (85) indicates that a degree of order and chaos can be associated with the gravitational field characterized by the 3-metric $g_{a b}$. More precisely, we can say that the density of particles $\rho=R^{2}$ associated with the wave functional $\Psi$ determines a modification of the gravitational space described by the quantum entropy for the gravitational field (85). With the introduction of the quantum entropy (85), the quantum potential for the gravitational field (82) can be expressed in the convenient regularized way:

$Q_{G}=\hbar^{2} N g\left[G_{a b c d}\left(\frac{\delta^{2}}{\delta g_{a b} \delta g_{c d}}\right)_{g} S_{Q}+\right.$

$\left.+g^{-q} \frac{\delta g^{q}}{\delta g_{a b}}\left(\frac{\delta}{\delta g_{c d}}\right)_{g} S_{Q}\right]$.

On the basis of Eq. (86), the quantum entropy for the gravitational field can be considered as the fundamental entity, as the ultimate visiting card, which determines the action of the quantum potential on the gravitational field. The following interesting perspective is therefore opened. In the non-relativistic de Broglie-Bohm theory, the quantum entropy represents the fundamental entity determining the behavior of subatomic particles. Analogously, in the Bohmian approach to the WDW equation, the quantum entropy for the gravitational field can be considered as the ultimate visiting card, which produces the behavior of the Universe in the presence of a gravitational field. And, in virtue of Eq. (86), we can say that Einstein's equations (83) and (84) state that the curvature of space is determined by the quantum entropy for the gravitational field.

\section{Conclusions}

In the de Broglie-Bohm theory, the quantum entropy - describing the degree of order and chaos of the back- 
ground space determined by the density of particles associated with the wave function under consideration turns out to be the fundamental entity, the ultimate visiting card, in the non-relativistic domain, in the relativistic curved space-time, and in the quantum gravity domain.

In the non-relativistic domain, it is just the quantum entropy as the fundamental element that determines the fact that the quantum potential acts as an active information channel on the behavior of the particles under consideration. The geometrodynamic nature of the quantum potential - namely, the fact that the quantum potential has a geometric nature contains a global information on the environment, in which the experiment is performed, and, at the same time, it is a dynamical entity - is determined by the background space described by the quantum entropy. The quantum entropy indicates what really are the geometric properties of space, from which the quantum force and, thus, the behavior of quantum particles are derived.

In the relativistic domain in a curved space-time, the quantum potential is the conformal degree of freedom of the space-time metric. Thus, the effects of gravity on geometry and the quantum effects on the geometry of space-time are highly coupled as a consequence of the quantum entropy, the background space described by the quantum entropy, and the degree of order and chaos of the vacuum supporting the density of particles associated with the wave function under consideration.

In the quantum gravity domain in the context of a scalar-tensor model suggested by F. Shojai and A. Shojai, the quantum entropy emerges as the fundamental entity, which produces the links between the quantum effects and the background and is, indeed, a dynamical factor. In particular, the causal structure and the scale factor of space-time are determined by the quantum entropy. Finally, as regards the Bohmian approach to the Wheeler-DeWitt equation, the perspective is opened that a quantum entropy for the gravitational field represents the ultimate visiting card, which determines the action of the quantum potential on the gravitational field and, thus, the behavior of the Universe in the presence of a gravitational field.

1. J. Von Neumann, Mathematical Foundations of Quantum Mechanics (Princeton Univ. Press, Princeton, 1955).

2. C.F. von Weizsäcker, in The Physicist's Conception of Nature, edited by J. Mehra (Reidel, Boston, 1973).

3. E. Schrödinger, Naturwiss. 23, 48, 807, 823, 844 (1935).
4. S. Bergia, in Quanti Copenaghen? Bohr, Heisenberg e le Interpretazioni della Meccanica Quantistica, edited by I. Tassani (Ponte Vecchio, Cesena, 2004), p. 179.

5. D. Fiscaletti, I Fondamenti della Meccanica Quantistica. Un'Analisi Critica dell'Interpretazione Ortodossa, della Teoria di Bohm e della Teoria GRW (CLEUP, Padova, 2003).

6. D. Fiscaletti, I Gatti di Schrödinger. Meccanica Quantistica e Visione del Mondo (Muzzio Editore, Roma, 2007).

7. P.R. Holland, The Quantum Theory of Motion (Cambridge Univ. Press, Cambridge, 1993).

8. D. Fiscaletti, Quant. Biosyst. 2, 93 (2007); www.quantumbionet.org.

9. L. de Broglie, J. de Phys. et Radium 8, 225 (1927).

10. L. de Broglie, in Solvay Congress (1927), Electrons and photons: rapports et discussions du Cinquime Conseil de Physique tenu Bruxelles du 24 au Octobre 1927 sous les auspices de l'Istitut International de Physique Solvay (Gauthier-Villars, Paris, 1928).

11. D. Bohm, Phys. Rev. 85, 166 (1952).

12. R.E. Wyatt, Quantum Dynamics with Trajectories: Introduction to Quantum Hydrodynamics (Springer, New York, 2005).

13. S. Goldstein, R. Tumulka, and N. Zanghì, arXiv:0912.2666v1 [quant-ph] (2009).

14. D. Bohm and B.J. Hiley, The Undivided Universe: an Ontological Interpretation of Quantum Theory (Routledge, London, 1993).

15. D. Fiscaletti, Ukr. J. Phys. 57, 560 (2012).

16. D. Bohm, in Symposium on the Foundation of Modern Physics - 1987, edited by P. Lahti and P. Mittelstaedt (World Scientific, Singapore, 1988).

17. M. Abolhasani and M. Golshani, Ann. de la Fondat. L. de Broglie 28, 1 (2003).

18. V.I. Sbitnev, Kvant. Magiya 5, 1101 (2008); URL http://quantmagic.narod.ru/volumes/VOL512008/ p1101.html.

19. L. Brillouin, Science and Information Theory (Dover, New York, 2004).

20. E.R. Bittner, http://arXiv.org/abs/quant-ph/0001119 (2000).

21. B. Poirier, http://arXiv.org/abs/0803.0193 (2008).

22. P.V. Poluyan, Kvant. Magiya, 2, 3119 (2005); http:// quantmagic.narod.ru/volumes/VOL232005/p3119.html.

23. V.I. Sbitnev, Int. J. of Bifurc. and Chaos 19, 2335 (2009); arXiv:0808.1245v1 [quant-ph] (2008).

24. C. Grosche, Path Integrals, Hyperbolic Spaces, and Selberg Trace Formulae (World Scientific, Singapore, 1996).

25. A. Shojai and F. Shojai, Phys. Scr. 64, 413 (2001).

26. F. Shojai and A. Shojai, arXiv:gr-qc/0404102 v1 (2004). 
27. L. de-Broglie, Non-Linear Wave Mechanics, (Elsevier, Amsterdam, 1960).

28. T. Horiguchi, Mod. Phys. Lett. A 9, 1429 (1994).

29. A. Blaut and J.K. Glikman, Class. Quant. Grav. 13, 39 (1996).

30. S.P. Kim, Phys. Lett. A 236, 11 (1997).

31. S.P. Kim, Phys. Rev. D 55, 7511 (1997).

32. Bohmiam Mechanics and Quantum Theory: An Appraisal, edited by J.T. Cushing, A. Fine, and S. Goldstein (Kluwer, Boston, 1996).

33. J.A. de Barros, N. Pinto-Neto, and M.A. Sagioro-Leal, Phys. Lett. A 241, 229 (1998).

34. F. Shojai and M. Golshani, Int. J. Mod. Phys. A. 13, 677 (1998)

35. R. Colistete, J.C. Fabris, and N. Pinto-Neto, Phys. Rev. D 57, 4707 (1998).

36. P. Pinto-Neto and R. Colistete, Phys. Lett. A 290, 219 (2001).

37. J. Marto and P.V. Moniz, Phys. Rev. D 65, 023516 (2001).

38. Kenmoku, R. Sato, and S. Uchida, Class. Quantum Grav. 19, 799 (2002)

39. F. Shojai, A. Shojai, and M. Golshani, Mod. Phys. Lett. A 13, 2725 (1998).

40. F. Shojai, A. Shojai, and M. Golshani, Mod. Phys. Lett. A., 13, 2915 (1998).

41. A. Shojai, F. Shojai, and M. Golshani, Mod. Phys. Lett. A 13, 2965 (1998).
42. F. Shojai and A. Shojai, Int. J. Mod. Phys. A 15, 1859 (2000).

43. A. Shojai, Int. J. Mod. Phys. A 15, 1757 (2000); arXiv:grqc/0010013.

44. F. Shojai and A. Shojai, arXiv: gr-qc/0306099 (2003).

45. Y.M. Cho and D.H. Park, Nuovo Cim. B 105, 817 (1990).

46. J. Kowalski-Glikman, arXiv:gr-qc/9511014 v1 (1995).

47. F. Shojai and A. Shojai, J. High En. Phys. 5, 037 (2001).

48. A. Shojai, F. Shojai, arXiv.gr-qc/0311076 v1 (2003).

49. A. Shojai and F. Shojai, Phys. Scr. 68, 207 (2003).

Received 09.10.11

КВАНТОВА ЕНТРОПІЯ В ГРАНИЧНОМУ ВИПАДКУ ТЕОРІЇ ДЕ БРОЙЛЯ-БОМА

Д. Фіскалетті

Р е з ю м е

Теорія де Бройля-Бома є цікавим напрямком у квантовій механіці, який описує атомні і субатомні процеси, не приписуючи особливої ролі спостерігачу і дотримуючись принципу причинності і постулату руху. У статті запропоновано нову змістовну інтерпретацію теорії де Бройля-Бома. Вона грунтується на ідеї, що квантова ентропія відноситься до їі граничного випадку в квантовій області, в релятивістському викривленому просторі-часі і в квантовій гравітації. 